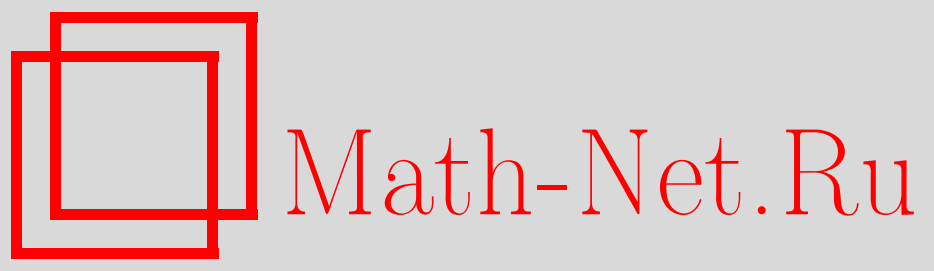

A. В. Перескоков, Асимптотика спектра оператора Хартри вблизи верхних границ спектральных кластеров. Асимптотические решения, локализованные вблизи окружности, ТМФ, 2015, том 183, номер 1, 78-89

DOI: https://doi.org/10.4213/tmf8761

Использование Общероссийского математического портала Math-Net.Ru подразумевает, что вы прочитали и согласны с пользовательским соглашением http://www . mathnet.ru/rus/agreement

Параметры загрузки:

IP : 54.198 .55 .26

26 апреля 2023 г., $17: 42: 59$

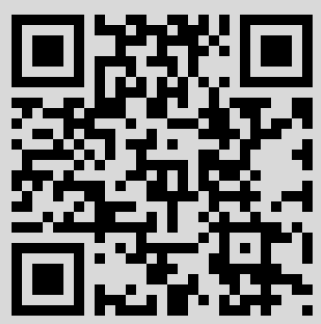




\title{
АСИМПТОТИКА СПЕКТРА ОПЕРАТОРА ХАРТРИ ВБЛИЗИ ВЕРХНИХ ГРАНИЦ СПЕКТРАЛЬНЫХ КЛАСТЕРОВ. АСИМПТОТИЧЕСКИЕ РЕШЕНИЯ, ЛОКАЛИЗОВАННЫЕ ВБЛИЗИ ОКРУЖНОСТИ
}

\begin{abstract}
Рассмотрена задача на собственные значения для оператора Хартри с малым параметром перед нелинейностью. Найдены асимптотические собственные значения и асимптотические собственные функции вблизи верхних границ спектральных кластеров, которые образуются около уровней энергии невозмущенного оператора. Вблизи окружности, где локализовано решение, главный член разложения является решением задачи о двумерном осцилляторе.
\end{abstract}

Ключевые слова: самосогласованное поле, спектральный кластер, асимптотические собственные значения и собственные функции, двумерный осциллятор, логарифмическая особенность.

DOI: $10.4213 / \operatorname{tmf} 8761$

\section{1. ВВЕДЕНИЕ}

Рассмотрим задачу на собственные значения для нелинейного оператора Хартри в пространстве $L^{2}\left(\mathbb{R}^{3}\right)$ с кулоновским взаимодействием:

$$
\left(-\Delta_{q}-\frac{1}{|q|}+\varepsilon \int_{\mathbb{R}^{3}} \frac{\left|\psi\left(q^{\prime}\right)\right|^{2}}{\left|q-q^{\prime}\right|} d q^{\prime}\right) \psi=\lambda \psi, \quad\|\psi\|_{L^{2}\left(\mathbb{R}^{3}\right)}=1,
$$

где $\Delta_{q}$ - оператор Лапласа, $\varepsilon>0$ - малый параметр. Уравнение самосогласованного поля во внешнем поле, содержащее интегральную нелинейность типа Хартри, играет фундаментальную роль в квантовой теории и нелинейной оптике. В частности, такие уравнения возникают в теории полярона, который можно рассматривать как простейший пример частицы, взаимодействующей с квантовым полем [1], [2], в теории конденсата Бозе-Эйнштейна [3], при нахождении электронных орбиталей

*Федеральное государственное бюджетное образовательное учреждение высшего профессионального образования Национальный исследовательский университет МЭИ, Москва, Россия. E-mail: pereskokov62@mail.ru

${ }^{\dagger}$ Национальный исследовательский университет Высшая школа экономики - Московский институт электроники и математики, Москва, Россия 
в многоэлектронных атомах [4], а также при рассмотрении сред с пространственной дисперсией [5].

Хорошо известно, что при $\varepsilon=0$ собственные значения $\lambda=\lambda_{n}(\varepsilon)$ задачи (1) равны

$$
\lambda_{n}(0)=-\frac{1}{4 n^{2}}, \quad n=1,2, \ldots
$$

Здесь $n$ - главное квантовое число. Для задачи (1) имеются теоремы существования, в частности для нижней точки спектра, которая отвечает основному состоянию [6], [7]. В работе [8] при $n=2$ было доказано существование состояний, не обладающих сферической симметрией, а также найдены пять ветвей собственных значений, выходящих из невозмущенной точки спектра.

Вопрос о существовании состояний, отличных от основного, является исходным при исследовании процессов, связанных с возбуждением электронов в поляронных средах [9]. В настоящее время, помимо чисто теоретического интереса, проблема возбужденных поляронных состояний вызывает интерес в связи с проблемой электронного переноса возбуждений в самых различных конденсированных средах. В частности, проблема электронного переноса на большие расстояния является одной из центральных в молекулярной биологии при описании коллективных возбуждений в молекулярных цепочках и в молекулах ДНК [10].

Рассмотрим случай, когда квантовое число $n$, задающее невозмущенное собственное значение, велико (для определенности будем считать, что $\lambda$ имеет порядок $\varepsilon$ ). Сформулируем основной результат.

Пусть $p=n-m-1$, где $m$ - магнитное квантовое число. В настоящей работе для каждого $p=0,1,2, \ldots$ найдены собственные значения

$$
\lambda_{n, i}^{(p)}(\varepsilon)=-\frac{1}{4 n^{2}}+\varepsilon\left(\frac{\ln n}{4 \pi n^{2}}+\frac{E_{1, i}^{(p)}}{n^{2}}\right)+O\left(\frac{\varepsilon \ln n}{n^{5 / 2}}\right), \quad n \rightarrow \infty,
$$

где $i=0,1, \ldots, I_{p}$, которые расположены вблизи верхних границ спектральных кластеров. Эти кластеры образуются вокруг уровней энергии (2) невозмущенного оператора $-\Delta_{q}-1 /|q|$ при добавлении к нему интегральной нелинейности Хартри. В частности, при $p=0,1,2$ числа $E_{1, i}^{(p)}$ представимы в виде

$$
E_{1, i}^{(p)}=\frac{1}{4 \pi}\left(5 \ln 2+\gamma-\frac{\sigma_{i}^{(p)}}{128}\right)
$$

где $\gamma \approx 0.57$ - постоянная Эйлера. При $p=0$ существует одно значение

$$
\sigma_{0}^{(0)}=0,
$$

при $p=1$ - два значения

$$
\sigma_{0}^{(1)}=80, \quad \sigma_{1}^{(1)}=96,
$$

а при $p=2$ - шесть значений

$$
\begin{aligned}
& \sigma_{0}^{(2)}=123+\frac{19}{39}, \quad \sigma_{1}^{(2)}=142, \quad \sigma_{2}^{(2)}=144, \\
& \sigma_{3}^{(2)}=145-\frac{1}{33}, \quad \sigma_{4}^{(2)}=145-\frac{1}{81}, \quad \sigma_{5}^{(2)}=147+\frac{5}{9} .
\end{aligned}
$$


Соответствующие (3) асимптотические собственные функции локализованы вблизи окружности Г в пространстве $\mathbb{R}^{3}$, на которой кулоновское ядро самодействия в (1) имеет логарифмическую особенность. Поэтому поправка в формуле (3) содержит $\ln n$, а числа $\lambda_{n, i}^{(p)}(\varepsilon)$ расположены вблизи верхних границ кластеров. Отметим, что на нижней границе кластера

$$
\lambda_{n}(\varepsilon) \sim-\frac{1}{4 n^{2}}+\frac{\varepsilon E_{\min }}{n^{2}}, \quad n \rightarrow \infty,
$$

где число $E_{\min }$ удовлетворяет неравенству

$$
E_{\min } \leqslant \frac{1}{2 \pi^{3}} \int_{0}^{\pi} \int_{0}^{\pi} K\left(\frac{\sqrt{\sin \theta \sin \theta^{\prime}}}{\sin \left(\left(\theta+\theta^{\prime}\right) / 2\right)}\right) \frac{d \theta^{\prime} d \theta}{\sin \left(\left(\theta+\theta^{\prime}\right) / 2\right)} .
$$

Здесь $K(\kappa)$ - полный эллиптический интеграл первого рода.

Теории квазиклассического приближения для уравнения Хартри, а также асимптотическим решениям уравнений типа Хартри, локализованным вблизи маломерных инвариантных подмногообразий в фазовом пространстве, посвящено большое число работ (см., например, [11]-[20]). В настоящей работе главный член асимптотического разложения вблизи окружности, где локализовано решение, оказывается решением другой классической задачи квантовой механики - задачи о двумерном осцилляторе:

$$
\mathbf{L} g_{0, i}^{(p)}(\tau, s)=0, \quad\left\|g_{0, i}^{(p)}\right\|_{L^{2}\left(\mathbb{R}^{2}\right)}=1 .
$$

Здесь оператор $\mathbf{L}$ задается как

$$
\mathbf{L}=-\frac{\partial^{2}}{\partial s^{2}}-\frac{\partial^{2}}{\partial \tau^{2}}+\left[s^{2}+\tau^{2}-2(p+1)\right] .
$$

В результате функция $g_{0, i}^{(p)}$ представима в виде линейной комбинации базисных собственных функций задачи (8), отвечающих собственному значению $p+1$. Коэффициенты разложения находятся из системы нелинейных уравнений одновременно с нахождением чисел $E_{1, i}^{(p)}$. Отметим, что эта система выводится из условий разрешимости уравнений для последующих приближений.

Аналогичные (1) задачи на собственные значения для возмущенного двумерного резонансного осциллятора, возбуждающий потенциал которого задается интегральной нелинейностью типа Хартри с гладким потенциалом самодействия, рассматривались ранее в работах [21]-[23]. В них в случае, когда потенциал самодействия является многочленом, были найдены асимптотические собственные значения и асимптотические собственные функции вблизи верхних границ спектральных кластеров.

Метод построения квазиклассических асимптотик в гладком случае основан на алгебраическом усреднении возмущения, последующем переходе в алгебру симметрий и когерентном преобразовании исходного представления этой алгебры в ее неприводимое представление в пространстве функций над лагранжевым подмногообразием в симплектическом листе [24]. Кроме того, при нахождении асимптотического решения использовалось новое интегральное представление. Если сравнить серии асимптотических собственных значений, полученные в работах [21]-[23], с собственными значениями (3), то они не содержат логарифмических поправок, а расщепление спектра в них происходит в следующем приближении. 


\section{2. ПОСТРОЕНИЕ АСИМПТОТИЧЕСКОГО РЕШЕНИЯ}

Пользуясь растяжением $q=x / \varepsilon, \psi=\varepsilon^{3 / 2} v, \lambda=\varepsilon E$, приведем задачу (1) $\mathrm{k}$ стандартному для теории квазиклассических приближений виду

$$
\left(-\varepsilon \Delta_{x}-\frac{1}{|x|}+\varepsilon \int_{\mathbb{R}^{3}} \frac{\left|v\left(x^{\prime}\right)\right|^{2}}{\left|x-x^{\prime}\right|} d x^{\prime}\right) v(x)=E v(x), \quad\|v\|_{L^{2}\left(\mathbb{R}^{3}\right)}=1 .
$$

Хорошо известно, что дискретным собственным значениям

$$
E_{n}=-\frac{1}{4 \varepsilon n^{2}}, \quad n=1,2, \ldots
$$

невозмущенной задачи

$$
\left(-\varepsilon \Delta_{x}-\frac{1}{|x|}\right) v(x)=E v(x), \quad\|v\|_{L^{2}\left(\mathbb{R}^{3}\right)}=1,
$$

в сферических координатах $(r, \theta, \varphi)$, где $0 \leqslant r \leqslant \infty, 0 \leqslant \theta \leqslant \pi, 0 \leqslant \varphi \leqslant 2 \pi$, отвечают собственные функции

$$
v_{n, k, n_{\mathrm{r}}}=Y_{\ell m}(\theta, \varphi) R_{n \ell}(r) .
$$

Здесь $\ell, n_{\mathrm{r}}-$ орбитальное и радиальное квантовые числа, $n=\ell+1+n_{\mathrm{r}}, k=\ell-m$, а функции $Y_{\ell m}$ и $R_{n \ell}$ выражаются через присоединенные полиномы Лежандра и полиномы Лагерра [25].

Пусть

$$
a=2 \ell^{2} \varepsilon,
$$

а число $\ell$ имеет порядок $\varepsilon^{-1 / 2}$. Введем новые переменные

$$
\tau=\left(\theta-\frac{\pi}{2}\right) \sqrt{\ell}, \quad s=\left(\frac{r}{a}-1\right) \sqrt{\ell} .
$$

Справедлива

Tеорема 1. При $\ell \rightarrow \infty$ и небольших $n_{\mathrm{r}}=0,1,2, \ldots u k=0,1,2, \ldots$ бункиии $v_{n, k, n_{\mathrm{r}}}$ по модулю $O\left(\ell^{-\infty}\right)$ сосредоточены вблизи окружности

$$
\Gamma_{a}=\{(r, \theta, \varphi) \mid r=a, \theta=\pi / 2\} \subset \mathbb{R}^{3} .
$$

Кроме того, при $s^{6}+\tau^{4} \ll \ell$ справедлива асимптотика

$$
\begin{aligned}
v_{n, k, n_{\mathrm{r}}}= & \frac{(-1)^{p} \sqrt{\ell}}{a^{3 / 2} 2^{(p+1) / 2} \pi \sqrt{n_{\mathrm{r}}} \cdot \sqrt{k !}} e^{i m \varphi} e^{-\left(s^{2}+\tau^{2}\right) / 2} H_{n_{\mathrm{r}}}(s) H_{k}(\tau) \times \\
& \times\left[1+O\left(\ell^{-1 / 2}\left(|s|^{3}+1\right) H_{n_{\mathrm{r}}}(s)\right)+O\left(\ell^{-1 / 2}\left(s^{2}+1\right) H_{n_{\mathrm{r}}}^{\prime}(s)\right)+\right. \\
& \left.\quad+O\left(\ell^{-1}\left(\tau^{4}+1\right) H_{k}(\tau)\right)+O\left(\ell^{-1}\left(|\tau|^{3}+1\right) H_{k}^{\prime}(\tau)\right)\right] .
\end{aligned}
$$

Здесъ $H_{n}-$ полином Эрмита.

Ниже для квантовых чисел $\ell, n, m$ порядка $\varepsilon^{-1 / 2}$ и, следовательно, для небольших чисел $n_{\mathrm{r}}, k$ и $p=n_{\mathrm{r}}+k$ построены асимптотические решения задачи $(10)$, по 
модулю $O\left(\ell^{-1}\right)$ локализованные вблизи окружности $\Gamma_{a}$. Переходя в сферическую систему координат, а также делая подстановку

$$
v(x)=\frac{e^{i m \varphi}}{\sqrt{2 \pi}} g(r, \theta),
$$

преобразуем задачу (10) к виду [18]

$$
\begin{gathered}
\left\{-\varepsilon\left[\frac{\partial^{2}}{\partial r^{2}}+\frac{2}{r} \frac{\partial}{\partial r}+\frac{1}{r^{2}}\left(\frac{\partial^{2}}{\partial \theta^{2}}+\operatorname{ctg} \theta \frac{\partial}{\partial \theta}-\frac{m^{2}}{\sin ^{2} \theta}\right)\right]-\frac{1}{r}+\right. \\
\left.+\varepsilon \int_{0}^{\pi} \int_{0}^{\infty} W\left(r, r^{\prime}, \theta, \theta^{\prime}\right)\left|g\left(r^{\prime}, \theta^{\prime}\right)\right|^{2}\left(r^{\prime}\right)^{2} \sin \theta^{\prime} d r^{\prime} d \theta^{\prime}-E\right\} g(r, \theta)=0, \\
\int_{0}^{\pi} \int_{0}^{\infty}|g(r, \theta)|^{2} r^{2} \sin \theta d r d \theta=1,
\end{gathered}
$$

где ядро имеет вид

$$
W\left(r, r^{\prime}, \theta, \theta^{\prime}\right)=\frac{2}{\pi \sqrt{r^{2}+\left(r^{\prime}\right)^{2}-2 r r^{\prime} \cos \left(\theta+\theta^{\prime}\right)}} K\left(\frac{2 \sqrt{r r^{\prime} \sin \theta \sin \theta^{\prime}}}{\sqrt{r^{2}+\left(r^{\prime}\right)^{2}-2 r r^{\prime} \cos \left(\theta+\theta^{\prime}\right)}}\right) .
$$

Асимптотические решения задачи (13), (14) будем искать в виде

$$
\begin{gathered}
g=a^{-3 / 2}\left[\sqrt{\ell} g_{0}(\tau, s)+g_{1}(\tau, s)+\frac{g_{2}(\tau, s)}{\sqrt{\ell}}\right]+O\left(\frac{g_{3}(\tau, s)}{\ell}\right), \\
E=-\frac{1}{2 a\left(1+\left(n_{\mathrm{r}}+1\right) / \ell\right)^{2}}+\frac{E_{0} \ln \ell}{\ell^{2}}+\frac{E_{1}}{\ell^{2}}+O\left(\frac{\ln \ell}{\ell^{5 / 2}}\right) .
\end{gathered}
$$

В этих формулах $\ell \rightarrow \infty$, функции $g_{j}(\tau, s), j=0,1,2,3$, экспоненциально убывают при $\tau^{2}+s^{2} \rightarrow \infty ; E_{0}, E_{1}$ - некоторые константы (для упрощения обозначений индексы $i$ и $p$ у $g_{0}$ и $E_{1}$ опущены).

Разложим входящие в соотношения (13), (14) функции с помощью формулы Тейлора по степеням $\tau$ и $s$. Поскольку функция $K(\kappa)$ имеет логарифмическую особенность при $\kappa \rightarrow 1[26]$,

$$
K(\kappa)=\ln \frac{4}{\sqrt{1-\kappa^{2}}}+O\left(\left(1-\kappa^{2}\right) \ln \left(1-\kappa^{2}\right)\right),
$$

непосредственно к $W$ формула Тейлора неприменима. Пусть $u=\left(\tau-\tau^{\prime}\right)^{2}+\left(s-s^{\prime}\right)^{2}$. Тогда из соотношений (15), (18) вытекает

ЛЕмма 1. При $\ell \rightarrow \infty, u \ll \ell$ имеет место асимптотика

$$
W\left(r, r^{\prime}, \theta, \theta^{\prime}\right)=\frac{1}{\pi a} \ln \frac{8 \sqrt{\ell}}{\sqrt{u}}+O\left(\frac{s+s^{\prime}}{\sqrt{\ell}} \ln \frac{\sqrt{\ell}}{\sqrt{u}}\right)+O\left(\frac{u}{\ell} \ln \frac{\sqrt{\ell}}{\sqrt{u}}\right) .
$$

Далее разложим $\left(1+\left(n_{\mathrm{r}}+1\right) / \ell\right)^{-2}$ по степеням $\ell$ и подставим асимптотики $(16)$, (17) в уравнения (13), (14). В силу формул (12), (17), (19), (14) для отсутствия в левой части (13) слагаемых порядка $\ell^{-2} \ln \ell$ достаточно положить $E_{0}=1 / 4 \pi$. Приравнивая к нулю слагаемые порядков $\ell^{-1}, \ell^{-3 / 2}$ и $\ell^{-2}$, получаем задачи для определения $g_{0}, g_{1}$ и $g_{2}$. Справедлива 
ЛЕмма 2. Функиия $g_{0}$ является решением задачи о двумерном осиилляторе,

$$
\begin{gathered}
\mathbf{L} g_{0}=0, \\
\int_{\mathbb{R}^{2}}\left|g_{0}\right|^{2} d \tau d s=1,
\end{gathered}
$$

а функиии $g_{1}$ и $g_{2}-$ решениями задач

$$
\begin{aligned}
\mathbf{L} g_{1} & =F_{1} \\
\int_{\mathbb{R}^{2}}\left(g_{0} \bar{g}_{1}+\bar{g}_{0} g_{1}\right) d \tau d s & =-2 \int_{\mathbb{R}^{2}} s\left|g_{0}\right|^{2} d \tau d s
\end{aligned}
$$

$u$

$$
\begin{gathered}
\mathbf{L} g_{2}=F_{2,1}+F_{2,2} \\
\int_{\mathbb{R}^{2}}\left(g_{0} \bar{g}_{2}+\bar{g}_{0} g_{2}\right) d \tau d s=-\int_{\mathbb{R}^{2}}\left[\left|g_{1}\right|^{2}+2 s\left(g_{0} \bar{g}_{1}+\bar{g}_{0} g_{1}\right)+\left(s^{2}-\tau^{2} / 2\right)\left|g_{0}\right|^{2}\right] d \tau d s
\end{gathered}
$$

Здесъ оператор $\mathbf{L}$ задан формулой $(9)$, а функиии $F_{1}, F_{2,1}, F_{2,2}$ имеют вид

$$
\begin{aligned}
F_{1}(\tau, s)= & 2\left[\frac{\partial g_{0}}{\partial s}-s \frac{\partial^{2} g_{0}}{\partial \tau^{2}}+\left(s^{3}-2 k s+s \tau^{2}\right) g_{0}\right] \\
F_{2,1}(\tau, s)= & -2 s \frac{\partial g_{0}}{\partial s}+3 s^{2} \frac{\partial^{2} g_{0}}{\partial \tau^{2}}-\tau \frac{\partial g_{0}}{\partial \tau}- \\
& -\left[\frac{2}{3} \tau^{4}-2 k \tau^{2}+k^{2}-6 k s^{2}+3 s^{2} \tau^{2}+3 s^{4}+3\left(n_{\mathrm{r}}+1\right)^{2}\right] g_{0}+ \\
& +2\left[\frac{\partial g_{1}}{\partial s}-s \frac{\partial^{2} g_{1}}{\partial \tau^{2}}+\left(s^{3}-2 k s+s \tau^{2}\right) g_{1}\right], \\
F_{2,2}(\tau, s)= & \frac{1}{2 \pi}\left(4 \pi E_{1}-6 \ln 2+\int_{\mathbb{R}^{2}} \ln \left(\left(\tau-\tau^{\prime}\right)^{2}+\left(s-s^{\prime}\right)^{2}\right)\left|g_{0}\left(\tau^{\prime}, s^{\prime}\right)\right|^{2} d \tau^{\prime} d s^{\prime}\right) g_{0} .
\end{aligned}
$$

Решениями уравнения (20) в пространстве $L^{2}\left(\mathbb{R}^{2}\right)$ являются собственные функции двумерного осциллятора, которые отвечают собственному значению $p+1$ (здесь $p=0,1,2, \ldots)$. Они образуют подпространство $\mathcal{H}_{p} \subset L^{2}\left(\mathbb{R}^{2}\right)$, ортонормированный базис в котором состоит из функций $\beta_{j, p-j}(\tau, s), j=0,1, \ldots, p$, где

$$
\beta_{j, i}(\tau, s)=\theta_{j, i} e^{-\left(s^{2}+\tau^{2}\right) / 2} H_{j}(s) H_{i}(\tau), \quad \theta_{j, i}=\frac{(-1)^{j+i}}{2^{(j+i) / 2} \sqrt{\pi} \sqrt{j !} \sqrt{i !}} .
$$

Следовательно,

$$
g_{0}=\sum_{j=0}^{p} c_{j} \beta_{j, p-j}
$$

где $c_{j}$ - некоторые константы, удовлетворяющие условию нормировки

$$
\sum_{j=0}^{p}\left|c_{j}\right|^{2}=1
$$


Они находятся из условий разрешимости для последующих приближений. Используя (20), преобразуем правую часть уравнения (21) к виду

$$
\begin{aligned}
F_{1}= & 2\left[s \frac{\partial^{2} g_{0}}{\partial s^{2}}+\frac{\partial g_{0}}{\partial s}+2 s\left(n_{\mathrm{r}}+1\right) g_{0}\right]= \\
= & 2 \sum_{j=0}^{p} c_{j} \theta_{j, p-j} e^{-\left(s^{2}+\tau^{2}\right) / 2} H_{p-j}(\tau)\left\{\frac{H_{j+3}(s)}{8}+\left(\frac{3-j}{4}+n_{\mathrm{r}}\right) H_{j+1}(s)+\right. \\
& \left.+j\left(-\frac{j}{2}+2 n_{\mathrm{r}}+2\right) H_{j-1}(s)+j(j-1)(j-2) H_{j-3}(s)\right\} .
\end{aligned}
$$

Поскольку выражение $(24)$ не содержит функций из $\mathcal{H}_{p}$, уравнение $(21)$ разрешимо. Его решение имеет вид

$$
g_{1}=-\frac{1}{3} \frac{\partial^{3} g_{0}}{\partial s^{3}}-2\left(n_{\mathrm{r}}+1\right) \frac{\partial g_{0}}{\partial s}+\sum_{j=0}^{p} c_{j}^{*} \beta_{j, p-j},
$$

где $c_{j}^{*}$ - некоторые константы.

Аналогично доказывается, что $F_{2,1}$ также не содержит функций из $\mathcal{H}_{p}$. Поэтому условия разрешимости уравнения (22) принимают вид

$$
\int_{\mathbb{R}^{2}} F_{2,2} \beta_{j, p-j} d \tau d s=0, \quad j=0, \ldots, p .
$$

При выполнении этих условий функция $g_{2} \in L^{2}\left(\mathbb{R}^{2}\right)$ может быть представлена в виде суммы ряда [27], [28]

$$
\begin{aligned}
g_{2} & =\sum_{\substack{j, i=0, j+i \neq p}}^{\infty} \frac{1}{2(j+i-p)} \times \\
& \times \int_{\mathbb{R}^{2}}\left(F_{2,1}\left(\tau^{\prime}, s^{\prime}\right)+a F_{2,2}\left(\tau^{\prime}, s^{\prime}\right)\right) \beta_{j, i}\left(\tau^{\prime}, s^{\prime}\right) d \tau^{\prime} d s^{\prime} \beta_{j, i}(\tau, s)+\sum_{j=0}^{p} c_{j}^{* *} \beta_{j, p-j},
\end{aligned}
$$

где $c_{j}^{* *}$ - некоторые константы.

Условия (25) позволяют найти входящие в (23) коэффициенты:

$c_{j}=\frac{1}{6 \ln 2-4 \pi E_{1}} \int_{\mathbb{R}^{4}} \ln \left(\left(\tau-\tau^{\prime}\right)^{2}+\left(s-s^{\prime}\right)^{2}\right)\left|g_{0}\left(\tau^{\prime}, s^{\prime}\right)\right|^{2} g_{0}(\tau, s) \beta_{j, p-j}(\tau, s) d \tau^{\prime} d s^{\prime} d \tau d s$, где $j=0, \ldots, p$. Справедлива

ЛЕмма 3. Функция $g_{0} \in \mathcal{H}_{p}$ и число $E_{1}$ являются решением следующей задачи на собственные значения:

$$
\begin{aligned}
& \left(6 \ln 2-4 \pi E_{1}\right) g_{0}= \\
& =\int_{\mathbb{R}^{2}} \Omega\left(\tau, s, \tau^{\prime \prime}, s^{\prime \prime}\right) \int_{\mathbb{R}^{2}} \ln \left(\left(\tau^{\prime}-\tau^{\prime \prime}\right)^{2}+\left(s^{\prime}-s^{\prime \prime}\right)^{2}\right)\left|g_{0}\left(\tau^{\prime}, s^{\prime}\right)\right|^{2} d \tau^{\prime} d s^{\prime} g_{0}\left(\tau^{\prime \prime}, s^{\prime \prime}\right) d \tau^{\prime \prime} d s^{\prime \prime},
\end{aligned}
$$




$$
\int_{\mathbb{R}^{2}}\left|g_{0}(\tau, s)\right|^{2} d \tau d s=1
$$

Здесъ

$$
\Omega\left(\tau, s, \tau^{\prime \prime}, s^{\prime \prime}\right)=\frac{e^{-\left(s^{2}+\tau^{2}+\left(s^{\prime \prime}\right)^{2}+\left(\tau^{\prime \prime}\right)^{2}\right) / 2}}{2^{p} \pi} \sum_{j=0}^{p} \frac{H_{j}(s) H_{j}\left(s^{\prime \prime}\right) H_{p-j}(\tau) H_{p-j}\left(\tau^{\prime \prime}\right)}{j !(p-j) !} .
$$

Из соотношений $(27),(28)$ следует, что число $E_{1}$ может быть представлено в виде

$$
\begin{aligned}
E_{1}= & \frac{3 \ln 2}{2 \pi}-\frac{1}{4 \pi} \int_{\mathbb{R}^{2}} \int_{\mathbb{R}^{2}} \Omega\left(\tau, s, \tau^{\prime \prime}, s^{\prime \prime}\right) g_{0}\left(\tau^{\prime \prime}, s^{\prime \prime}\right) \bar{g}_{0}(\tau, s) d \tau d s \times \\
& \times \int_{\mathbb{R}^{2}} \ln \left(\left(\tau^{\prime}-\tau^{\prime \prime}\right)^{2}+\left(s^{\prime}-s^{\prime \prime}\right)^{2}\right)\left|g_{0}\left(\tau^{\prime}, s^{\prime}\right)\right|^{2} d \tau^{\prime} d s^{\prime} d \tau^{\prime \prime} d s^{\prime \prime} .
\end{aligned}
$$

ЗАмЕчАниЕ 1. Уравнение (27) является нелинейным интегральным уравнением. Ранее нелинейное интегральное уравнение на сфере возникало в работе [15]. Оно использовалось для нахождении серии асимптотических собственных функций оператора Хартри.

ЗАмЕчАниЕ 2. Для ядра $\Omega$ имеет место интегральное представление

$$
\begin{aligned}
\Omega\left(\tau, s, \tau^{\prime \prime}, s^{\prime \prime}\right)= & \frac{(-1)^{p} 2^{p}}{p ! \pi^{3}} e^{-\left(s^{2}+\tau^{2}+\left(s^{\prime \prime}\right)^{2}+\left(\tau^{\prime \prime}\right)^{2}\right) / 2} \times \\
& \times \int_{\mathbb{R}^{4}}\left(\tilde{s} \tilde{s}^{\prime}+\tilde{\tau} \tilde{\tau}^{\prime}\right)^{p} e^{(i \tilde{s}-s)^{2}+(i \tilde{\tau}-\tau)^{2}+\left(i \tilde{s}^{\prime}-s^{\prime}\right)^{2}+\left(i \tilde{\tau}^{\prime}-\tau^{\prime}\right)^{2}} d \tilde{\tau} d \tilde{s} d \tilde{\tau}^{\prime} d \tilde{s}^{\prime} .
\end{aligned}
$$

Пусть при некотором $p=0,1,2, \ldots$ число $E_{1}=E_{1, i}^{(p)}$ и функция $g_{0}=g_{0, i}^{(p)}$ являются решением задачи $(27),(28)$. Определим число $\lambda_{n, i}^{(p)}$ по формуле (3), а также функцию

$$
\psi_{n, i}^{(p)}=\varepsilon^{3 / 2} \sum_{j=0}^{p} c_{j} v_{n, p-j, j}(\varepsilon q)
$$

где $v_{n, k, n_{\mathrm{r}}}$ задается формулой $(11)$, коэффициенты $c_{j}=c_{j, i}^{(p)}$ совпадают с коэффициентами разложения функции $g_{0}$ согласно равенству (23) (см. теорему 1). Отметим, что в отличие от $g_{0}$ функция $\psi_{n, i}^{(p)}$ выражается через присоединенные полиномы Лежандра от $\cos \theta$ и, следовательно, удовлетворяет граничным условиям по $\theta$. Справедлива

ТЕОРема 2. При $\varepsilon \rightarrow 0$ u п порядка $\varepsilon^{-1 / 2}$ число $\lambda_{n, i}^{(p)}$ является асимтотическим собственным значением, а функция $\psi_{n, i}^{(p)}$ - главным членом разложения соответствующей собственной функиии задачи (1) в пространстве $L^{2}\left(\mathbb{R}^{3}\right)$. Число $\lambda_{n, i}^{(p)}$ расположено вблизи верхней гранищы спектрального кластера, образующегося вокруг числа (2), а функиия $\psi_{n, i}^{(p)}$ по модулю $O(n)$ локализована вблизи окружности

$$
\Gamma=\left\{(r, \theta, \varphi) \mid r=2 n^{2}, \theta=\pi / 2\right\} \subset \mathbb{R}^{3} .
$$

ЗАмЕчАниЕ 3. Поправка к $\psi_{n, i}^{(p)}$ строится аналогично (26), однако имеет весьма громоздкий вид. 


\section{3. ПРИМЕРЫ РЕШЕНИЯ СПЕКТРАЛЬНОЙ ЗАДАЧИ НА ПОДПРОСТРАНСТВАХ $\mathcal{H}_{P}$}

Найдем решение задачи $(27),(28)$ при $p=0$. Будем искать $g_{0,0}^{(0)}$ в виде

$$
g_{0,0}^{(0)}=c_{0}^{(0)} \beta_{0,0} .
$$

В силу условия нормировки (28) константа $c_{0}^{(0)}$ удовлетворяет равенству

$$
c_{0}^{(0)}=1 .
$$

ЗАмЕчание 4. Формулы для асимптотических собственных функций в настоящей статье приводятся с точностью до произвольного множителя вида $e^{i \varphi}$, где $\varphi \in \mathbb{R}$.

Подставляя (30) в соотношение (29), имеем

$$
\begin{aligned}
E_{1,0}^{(0)} & =\frac{3 \ln 2}{2 \pi}-\frac{1}{4 \pi^{3}} \int_{\mathbb{R}^{4}} \ln \left(\left(\tau^{\prime}-\tau^{\prime \prime}\right)^{2}+\left(s^{\prime}-s^{\prime \prime}\right)^{2}\right) e^{-s^{\prime 2}-\tau^{\prime 2}-\left(s^{\prime \prime}\right)^{2}-\left(\tau^{\prime \prime}\right)^{2}} d \tau^{\prime} d s^{\prime} d \tau^{\prime \prime} d s^{\prime \prime}= \\
& =\frac{5 \ln 2+\gamma}{4 \pi} .
\end{aligned}
$$

Напомним, $\gamma$ - постоянная Эйлера.

При $p=1$ решение будем искать в виде

$$
g_{0, i}^{(1)}=c_{0, i}^{(1)} \beta_{0,1}+c_{1, i}^{(1)} \beta_{1,0},
$$

где $c_{0, i}^{(1)}, c_{1, i}^{(1)}$ - константы. Вычисляя входящие в $(27),(28)$ интегралы, приходим к системе уравнений (индекс $i$ для краткости обозначений опущен)

$$
\begin{gathered}
\left(4 \pi E_{1}^{(1)}-5 \ln 2-\gamma+\frac{5}{8}\right) c_{0}^{(1)}+\left(c_{0}^{(1)} \bar{c}_{1}^{(1)}-\bar{c}_{0}^{(1)} c_{1}^{(1)}\right) \frac{c_{1}^{(1)}}{8}=0 \\
\left(4 \pi E_{1}^{(1)}-5 \ln 2-\gamma+\frac{5}{8}\right) c_{1}^{(1)}-\left(c_{0}^{(1)} \bar{c}_{1}^{(1)}-\bar{c}_{0}^{(1)} c_{1}^{(1)}\right) \frac{c_{0}^{(1)}}{8}=0 \\
\left|c_{0}^{(1)}\right|^{2}+\left|c_{1}^{(1)}\right|^{2}=1 .
\end{gathered}
$$

Данная система при

$$
E_{1,0}^{(1)}=\frac{1}{4 \pi}\left(5 \ln 2+\gamma-\frac{5}{8}\right)
$$

имеет однопараметрическое семейство вещественных решений

$$
c_{0,0}^{(1)}=\cos \alpha, \quad c_{1,0}^{(1)}=\sin \alpha, \quad \alpha \in \mathbb{R},
$$

а при

$$
E_{1,1}^{(1)}=\frac{1}{4 \pi}\left(5 \ln 2+\gamma-\frac{3}{4}\right)
$$

- комплексные решения

$$
c_{0,1}^{(1)}=\frac{1}{\sqrt{2}}, \quad c_{1,1}^{(1)}= \pm \frac{i}{\sqrt{2}} .
$$


Наконец, при $p=2$ будем искать решение задачи $(27),(28)$ в виде

$$
g_{0, i}^{(2)}=c_{0, i}^{(2)} \beta_{0,2}+c_{1, i}^{(2)} \beta_{1,1}+c_{2, i}^{(2)} \beta_{2,0} .
$$

Значительно более громоздкие вычисления приводят к следующей нелинейной системе:

$$
\begin{aligned}
& -\sigma^{(2)} c_{0}^{(2)}+\left|c_{0}^{(2)}\right|^{2}\left(\frac{247}{2} c_{0}^{(2)}+\frac{c_{2}^{(2)}}{2}\right)+\left|c_{1}^{(2)}\right|^{2}\left(153 c_{0}^{(2)}-c_{2}^{(2)}\right)+ \\
& +\left|c_{2}^{(2)}\right|^{2}\left(\frac{343}{2} c_{0}^{(2)}+\frac{c_{2}^{(2)}}{2}\right)-15\left(c_{0}^{(2)} \bar{c}_{1}^{(2)}+\bar{c}_{0}^{(2)} c_{1}^{(2)}\right) c_{1}^{(2)}- \\
& -9\left(c_{2}^{(2)} \bar{c}_{1}^{(2)}+\bar{c}_{2}^{(2)} c_{1}^{(2)}\right) c_{1}^{(2)}+\left(c_{0}^{(2)} \bar{c}_{2}^{(2)}+\bar{c}_{0}^{(2)} c_{2}^{(2)}\right)\left(\frac{c_{0}^{(2)}}{2}-\frac{9}{2} c_{2}^{(2)}\right)=0 \\
& -\sigma^{(2)} c_{1}^{(2)}+153\left|c_{0}^{(2)}\right|^{2} c_{1}^{(2)}+142\left|c_{1}^{(2)}\right|^{2} c_{1}^{(2)}+153\left|c_{2}^{(2)}\right|^{2} c_{1}^{(2)}- \\
& -\left(c_{0}^{(2)} \bar{c}_{2}^{(2)}+\bar{c}_{0}^{(2)} c_{2}^{(2)}\right) c_{1}^{(2)}-\left(c_{0}^{(2)} \bar{c}_{1}^{(2)}+\bar{c}_{0}^{(2)} c_{1}^{(2)}\right)\left(15 c_{0}^{(2)}+9 c_{2}^{(2)}\right)- \\
& -\sigma^{(2)} c_{2}^{(2)}+\mid c_{0}^{(2)} \bar{c}_{1}^{2}\left(\frac{c_{0}^{(2)}}{2}+\frac{343}{2} c_{2}^{(2)} c_{1}^{(2)}\right)\left(9 c_{0}^{(2)}+\left|c_{1}^{(2)}\right|^{2}\left(-c_{0}^{(2)}+15 c_{2}^{(2)}\right)=0\right. \\
& +\left|c_{2}^{(2)}\right|^{2}\left(\frac{c_{0}^{(2)}}{2}+\frac{247}{2} c_{2}^{(2)}\right)-9\left(c_{0}^{(2)}\right)+ \\
& -15\left(c_{2}^{(2)} \bar{c}_{1}^{(2)}+\bar{c}_{0}^{(2)} c_{1}^{(2)}\right) c_{1}^{(2)}- \\
& \left|c_{0}^{(2)}\right|^{2}+\left|c_{1}^{(2)}\right|^{2}+\left|c_{2}^{(2)}\right|^{2}=1
\end{aligned}
$$

где $\sigma^{(2)}=128\left(-4 \pi E_{1}^{(2)}+5 \ln 2+\gamma\right)$, а индекс $i$ снова опущен. Данная система имеет собственные значения $E_{1, i}^{(2)}, i=0,1,2,3,4,5$. Они задаются формулами (4), (7). Числам $E_{1,0}^{(2)}, E_{1,1}^{(2)}$ отвечают однопараметрические семейства решений $(35)$, коэффициенты которых имеют вид

$$
\begin{gathered}
c_{0,0}^{(2)}=\frac{1}{\sqrt{39}}\left(\frac{\sqrt{38}}{2}+\sqrt{10} \cos \alpha\right), \\
c_{1,0}^{(2)}=\frac{2 \sqrt{5}}{\sqrt{39}} \sin \alpha, \quad c_{2,0}^{(2)}=\frac{1}{\sqrt{39}}\left(\frac{\sqrt{38}}{2}-\sqrt{10} \cos \alpha\right), \\
c_{0,1}^{(2)}=\frac{\sin \alpha}{\sqrt{2}}, \quad c_{1,1}^{(2)}=\cos \alpha, \quad c_{2,1}^{(2)}=-\frac{\sin \alpha}{\sqrt{2}} .
\end{gathered}
$$

Здесь $\alpha \in \mathbb{R}$. Для $E_{1,2}^{(2)}$ имеется вещественное решение с коэффициентами

$$
c_{0,2}^{(2)}=\frac{1}{\sqrt{2}}, \quad c_{1,2}^{(2)}=0, \quad c_{2,2}^{(2)}=\frac{1}{\sqrt{2}} .
$$


Наконец, для $E_{1,3}^{(2)}, E_{1,4}^{(2)}, E_{1,5}^{(2)}$ существуют комплексные решения $g_{0, i}^{(2)}$, коэффициенты которых таковы: при $j=3$

$$
\begin{array}{rlrl}
c_{0,3}^{(2)} & =\frac{\sqrt{10} \pm \sqrt{8}}{2 \sqrt{33}} i, & c_{1,3}^{(2)}=\frac{\sqrt{8}}{\sqrt{11}}, & c_{2,3}^{(2)}=\frac{\sqrt{10} \mp \sqrt{8}}{2 \sqrt{33}} i, \\
c_{0,3}^{(2)}=-\frac{\sqrt{10} \pm \sqrt{8}}{2 \sqrt{33}} i, & c_{1,3}^{(2)}=\frac{\sqrt{8}}{\sqrt{11}}, & c_{2,3}^{(2)}=-\frac{\sqrt{10} \mp \sqrt{8}}{2 \sqrt{33}} i, \\
c_{0,3}^{(2)}=\frac{ \pm \sqrt{5}+\sqrt{24} i}{\sqrt{66}}, & c_{1,3}^{(2)}=\frac{\sqrt{8}}{\sqrt{66}}, & c_{2,3}^{(2)}=\frac{ \pm \sqrt{5}-\sqrt{24} i}{\sqrt{66}}, \\
c_{0,3}^{(2)}=\frac{ \pm \sqrt{5}-\sqrt{24} i}{\sqrt{66}}, & c_{1,3}^{(2)}=\frac{\sqrt{8}}{\sqrt{66}}, & c_{2,3}^{(2)}=\frac{ \pm \sqrt{5}+\sqrt{24} i}{\sqrt{66}} ;
\end{array}
$$

при $j=4$

$$
\begin{aligned}
& c_{0,4}^{(2)}= \pm \frac{\sqrt{22} i}{9}, \quad c_{1,4}^{(2)}=\frac{\sqrt{59}}{9}, \quad c_{2,4}^{(2)}=0, \\
& c_{0,4}^{(2)}=0, \quad c_{1,4}^{(2)}=\frac{\sqrt{59}}{9}, \quad c_{2,4}^{(2)}= \pm \frac{\sqrt{22} i}{9} ;
\end{aligned}
$$

при $j=5$

$$
\begin{aligned}
& c_{0,5}^{(2)}=\frac{1 \pm 4 \sqrt{5} i}{9 \sqrt{2}}, \quad c_{1,5}^{(2)}=0, \quad c_{2,5}^{(2)}=\frac{1}{\sqrt{2}}, \\
& c_{0,5}^{(2)}= \pm \frac{\sqrt{5} i}{3 \sqrt{2}}, \quad c_{1,5}^{(2)}=\frac{2}{3}, \quad c_{2,5}^{(2)}= \pm \frac{\sqrt{5} i}{3 \sqrt{2}} \text {. }
\end{aligned}
$$

Справедлива

ТЕОРема 3. При $p=0,1,2$ собственные значения задачи (27), (28) имеют вид, заданный формулами (4)-(7), а соответствующие собственные функции при $p=0$ определяются равенствами (30), (31), при р = 1 - равенствами (32) и (33), (34), а при р = 2 - равенствами (35) и (36)-(40).

Таким образом, мы нашли асимптотические собственные значения и асимптотические собственные функции для оператора Хартри вблизи верхних границ спектральных кластеров. Отметим, что использованные в работе методы носят общий характер. Они применимы не только в случае оператора Хартри, но и при изучении более сложных нелинейных уравнений с сингулярными ядрами.

Благодарности. Автор благодарен М.В. Карасеву за привлечение внимания к данной задаче, а также за ценные вопросы и замечания. Исследование выполнено за счет гранта Российского научного фонда (проект № 14-11-00306) и при частичной финансовой поддержке Программы поддержки ведущих научных школ (грант НШ-2081.2014.1).

\section{Список литературы}

[1] N. N. Bogolyubov, Украинск. матем. журн., 2:2 (1950), 3-24.

[2] С. И. Пекар, Исследования по электронной теории кристаллов, Гостехиздат, М., 1951.

[3] Л. П. Питаевский, УФН, 168:6 (1998), 641-653.

[4] Д.Р. Хартри, Расчеты атомных структур, ИЛ, М., 1960. 
[5] S. A. Achmanov, R. V. Hocklov, A. P. Suchorukov, "Self-defocusing and self-modulation in nonlinear media", Laserhandbuch, 2, North-Holland, Amsterdam, 1972, 5-108.

[6] E. H. Lieb, B. Simon, Commun. Math. Phys., 53:3 (1977), 185-194.

[7] P. L. Lions, Commun. Math. Phys., 109:1 (1987), 33-97.

[8] М. В. Карасев, Ю. В. Осипов, ТМФ, 52:2 (1982), 263-269.

[9] V.D. Lakhno, Возбужденные поляронные состояния в конденсированных средах, ОНТИ НЦБИ АН СССР, Пущино, 1990.

[10] А. С. Давыдов, Солитоны в молекулярных системах, Наукова думка, Киев, 1984.

[11] М. В. Карасев, В.П. Маслов, Итоги науки и техн. Сер. Соврем. пробл. матем., 13 (1979), 145-267.

[12] С. И. Черных, ТМФ, 52:3 (1982), 491-494.

[13] В. П. Маслов, Комплексный метод ВКБ в нелинейных уравнениях, Наука, М., 1977.

[14] И. В. Сименог, ТМФ, 30:3 (1977), 408-414.

[15] М. В. Карасев, Квантовая редукиия на орбиты алгебр симметрий и задача Эренфеста, Препринт ИТФ-87-157Р, ИТФ АН УССР, Киев, 1987.

[16] С. А. Вакуленко, В. П. Маслов, И. А. Молотков, И. А. Шафаревич, Докл. РАН, 345:6 (1995), 743-745.

[17] М. В. Карасев, А. В. Перескоков, Изв. РАН. Сер. матем., 65:5 (2001), 33-72.

[18] М. В. Карасев, А. В. Перескоков, Изв. РАН. Сер. матем., 65:6 (2001), 57-98.

[19] А. В. Перескоков, ТМФ, 131:3 (2002), 389-406.

[20] В. В. Белов, Ф. Н. Литвинец, А. Ю. Трифонов, ТМФ, 150:1 (2007), 26-40.

[21] А. В. Перескоков, ТМФ, 178:1 (2014), 88-106.

[22] А. В. Перескоков, Наноструктуры. Матем. физ. и моделирование, 10:1 (2014), 77-112.

[23] А. В. Перескоков, Вестн. МЭИ, 2013, № 6, 180-190.

[24] M. V. Karasev, "Noncommutative algebras, nano-structures, and quantum dynamics generated by resonances. I", Quantum Algebras and Poisson Geometry in Mathematical Physics, American Mathematical Society Translation Series 2, 216, AMS, Providence, RI, 2005, 1-17; Adv. Stud. Contemp. Math., 11:1 (2005), 33-56; Russ. J. Math. Phis., 13:2 (2006), $131-150$.

[25] Л. Шифф, Квантовая механика, ИЛ, М., 1957.

[26] Г. Бейтмен, А. Эрдейи, Высшие трансцендентные функиии, т. 3: Эллиптические и автоморфные функиии, функиии Ламе и Матье, Наука, М., 1967.

[27] Г. Сегё, Ортогональные многочлены, Физматлит, М., 1962.

[28] Г. Бейтмен, А. Эрдейи, Высшие трансцендентные функиии, т. 2: Функиии Бесселя, функции параболического ицлиндра, ортогональные многочлены, Наука, М., 1974.

Поступила в редакцию 1.07.2014, после доработки 25.09.2014 